\title{
OBTENCIÓN, ELECTRODEPOSICIÓN Y CARACTERIZACIÓN DE UN RECUBRIMIENTO POLIMÉRICO BIOABSORBIBLE A PARTIR DE ÁCIDO L-LÁCTICO PARA APLICACIONES BIOMÉDICAS
}

\section{SYNTHESIS, ELECTRODEPOSITION AND CHARACTERIZATION OF A BIOABSORBABLE POLYMER COATING FROM L-LACTIC ACID FOR BIOMEDICAL APPLICATIONS}

\author{
Diana Carolina Parada Quinayá1 $\quad$ Hugo Armando Estupiñán Durán ${ }^{1}$ \\ Darío Yesid Peña Ballesteros $^{1} \quad$ Custodio Vásquez Quintero $^{1}$ Dionisio Laverde Cataño ${ }^{1}$ \\ Recibido 6 de mayo de 2008, aceptado 9 de octubre de 2009 \\ Received: May 6, 2008 Accepted: October 9, 2009
}

\begin{abstract}
RESUMEN
El ácido poli (L-láctico) (APL) ha sido usado ampliamente en aplicaciones biomédicas como suturas e implantes bioabsorbibles debido a su capacidad para permitir el crecimiento controlado de tejido biológico durante su biodegradación controlada. En este trabajo se obtuvo APL a partir de la policondensación de ácido láctico en presencia de un catalizador de zinc metálico en condiciones de vacío y atmósfera inerte. Se variaron las concentraciones de $\mathrm{Zn}^{++}$y el tiempo de polimerización con el fin de obtener varias muestras que fueron caracterizadas mediante espectroscopia infrarroja (IR), calorimetría diferencial de barrido (DSC), análisis termogravimétrico (TGA) y microscopia electrónica de barrido (SEM). Se obtuvieron recubrimientos poliméricos por electrodeposición catódica sobre sustratos de Ti6Al4V, usando una celda electrolítica con capacidad de $200 \mathrm{ml}$ y dos ánodos de grafito cuya área expuesta fue de $12,57 \mathrm{~cm}^{2}$. El electrolito consistió en una disolución 30:70 v/v de APL y acetona. Las variables involucradas fueron: voltaje, tiempo, temperatura, velocidad de agitación y las condiciones de preparación del polímero. Los recubrimientos obtenidos se caracterizaron mediante microscopia óptica y ataque químico. Los resultados revelaron la obtención de APL y la influencia del iniciador de $\mathrm{Zn}^{++}$, el cual promovió la migración y formación de grupos catiónicos que lograron neutralizarse y condensarse sobre Ti6Al4V bajo cargas catódicas. El análisis térmico mostró que en el rango de temperatura, entre $25{ }^{\circ} \mathrm{C}$ - $50{ }^{\circ} \mathrm{C}$, no hubo variaciones importantes en la degradación térmica, indicando que el material podría ser utilizado para recubrir implantes ortopédicos.
\end{abstract}

Palabras clave: Ácido poli (L-láctico), electrodeposición, policondensación, polímeros bioabsorbibles, recubrimientos poliméricos.

\begin{abstract}
Poli (L-lactic) (PLA) has been used broadly in biomedical applications, such as sutures and bioadsorbable implant due to its capacity to allow the controlled growth of the biological tissue during their controlled biodegradation. In this work, a material polymeric PLA starting from the polycondensation in vacuum and inert atmosphere of lactic acid in presence of a catalyst of metallic zinc was obtained. In order to obtain different samples, both the Zn catalyst content and time of polycondensation were varied. Analysis of Infrared Spectroscopy, TGA, DSC, SEM and chemical etching were used to characterize the samples. Through a method of cathodic electrodeposition, a polymeric film was obtained on Ti6Al4V, by using an electrolytic cell with capacity of $200 \mathrm{ml}$ and two graphite anodes whose exposed area was $12,57 \mathrm{~cm}^{2}$. The electrolyte was a PLA-acetone dissolution 30:70 v v. For this process, the variables were: voltage, time, temperature, stirring and the preparation conditions of polymer. Optical microscopy and etching techniques were used to characterize the resulting polymeric film. It was evidenced the PLA formation using a metallic initiator $Z n$ which has provided migration and formation of cationic groups. These were efficiently neutralized and clustered on the Ti6Al4V substrate due to cathodic current application. In a range between 25 to $50{ }^{\circ} \mathrm{C}$, considerable variations of thermal degradation were observed which induces that the material can potentially be used as a coating in orthopedic implantations.
\end{abstract}

Keywords: Poly (L-lactic) acid, electrodeposition, polycondensation, bioabsorbables polymers, coatings polymers. 1 Grupo de Investigaciones en Corrosión. Universidad Industrial de Santander. Sede Guatiguará km 7, vía el Refugio, Piedecuesta, Santander, Colombia.
E-mail: icorros@ uis.edu.co; dypena@ uis.edu.co 


\section{INTRODUCCIÓN}

En las últimas décadas, el interés en el campo de los biomateriales [1] destinados a la regeneración ósea [2-4] $\mathrm{y}$ al desarrollo de materiales funcionales e inteligentes, capaces de estimular la respuesta biológica necesaria para restablecer las funciones que el tejido ha perdido [5], se encuentra en aumento. Entre los biomateriales más empleados para la fabricación de prótesis se encuentran el titanio y sus aleaciones [6], los cuales se han empleado satisfactoriamente en la implantación de prótesis dentales y de cirugía traumática.

Sin embargo, en el complejo organismo humano es necesario el aporte de materiales con otras propiedades muy diferentes, como son sistemas de gran flexibilidad y resistencia a la fatiga, aplicados a reemplazos de epidermis, tejido conectivo, sistema cardiovascular, sistema ocular, sistema digestivo, etc. Esto ha llevado a la búsqueda de nuevas alternativas en recubrimientos adecuados para estos materiales metálicos; recubrimientos que sean capaces de soportar las cargas iniciales posteriores a la implantación y que se degraden de forma controlada, transfiriendo las cargas de forma progresiva al nuevo hueso para poder ser reemplazado paulatinamente por el nuevo tejido [7-11].

Existen dos argumentos que favorecen el uso de materiales bioabsorbibles para estos recubrimientos; por una parte, son materiales que una vez concluida su función, desaparecen; y por otra, al desaparecer, permiten la regeneración de los tejidos donde se han implantado.

Polímeros como los obtenidos a partir del ácido láctico [11-17] pueden cumplir esta función temporal, abriendo la posibilidad de obtener un sinnúmero de aplicaciones, entre ellas, mejorar la adaptación de los implantes metálicos para recortar el tiempo de oseointegración, evitando una segunda intervención quirúrgica para la extracción del implante o funcionar como sistemas de liberación controlada de medicamentos.

En este trabajo se obtuvo ácido poli L-láctico a partir de la policondensación del ácido L-láctico en presencia de un catalizador de zinc metálico, el cual sirvió además para promover la presencia de grupos catiónicos en la electrodeposición. También se logró por un método de electrodeposición polimérica catódica [18] un recubrimiento del ácido poliláctico obtenido sobre sustratos de una aleación de Ti6Al4V.

El análisis de resultados reveló que la preparación de la emulsión con un iniciador de zinc metálico sobre el ácido L-láctico promueve la migración y formación de grupos catiónicos que logran neutralizarse y precipitarse sobre la superficie de los sustratos de Ti6Al4V bajo una carga catódica [19]. El recubrimiento polimérico obtenido fue caracterizado en función de algunas variables de electrodeposición.

Los avances alcanzados en este proyecto constituyen los primeros pasos en un estudio importante acerca de los materiales bioabsorbibles implantables y sobre recubrimientos con propiedades mejoradas para estos dispositivos, ya que el desarrollo de este procedimiento deja una ventana abierta para una investigación más profunda en este campo.

\section{EXPERIMENTACIÓN}

Los sustratos empleados suministrados por Quirúrgicos Especializados S.A. fueron discos de Ti6Al4V con $12 \mathrm{~mm}$ de diámetro y $2 \mathrm{~mm}$ de espesor (área de $3,02 \mathrm{~cm}^{2}$ ), los cuales fueron preparados superficialmente con papel abrasivo desde número 240 hasta 1200, limpiados en baño ultrasónico con acetona, neutralizados con agua destilada, secados y pesados.

En la síntesis del polímero, el monómero utilizado fue ácido L-láctico Carlo Erba al 98\%. El catalizador fue zinc de alta pureza Carlo Erba al $96 \%$ y acetona como solvente. La electrodeposición polimérica se llevó a cabo utilizando una fuente de potencia de $2.5 \mathrm{~A}$ y $500 \mathrm{~V}$ de corriente directa, electrodos de referencia para ensayos electroquímicos de grafito y un multímetro.

Para obtener mayor información sobre las variables involucradas en la obtención de ácido poliláctico a partir de ácido láctico por el método propuesto, se realizaron algunas pruebas preliminares, con el fin de obtener un material que pudiera ser comparado con el ácido poliláctico reportado en otras investigaciones.

Se realizaron además pruebas preliminares de electrodeposición catódica del poliláctico obtenido sobre los sustratos de Ti6Al4V, para analizar el efecto de las variables involucradas en el procedimiento de obtención del recubrimiento. Se llevaron a cabo las siguientes etapas:

- Síntesis de ácido poliláctico a partir de ácido láctico

- Caracterización del ácido poliláctico obtenido

- Electrodeposición del polímero sobre probetas Ti6Al4V

- Caracterización del recubrimiento obtenido.

La Figura 1 muestra un diagrama de las etapas desarrolladas durante el procedimiento. 


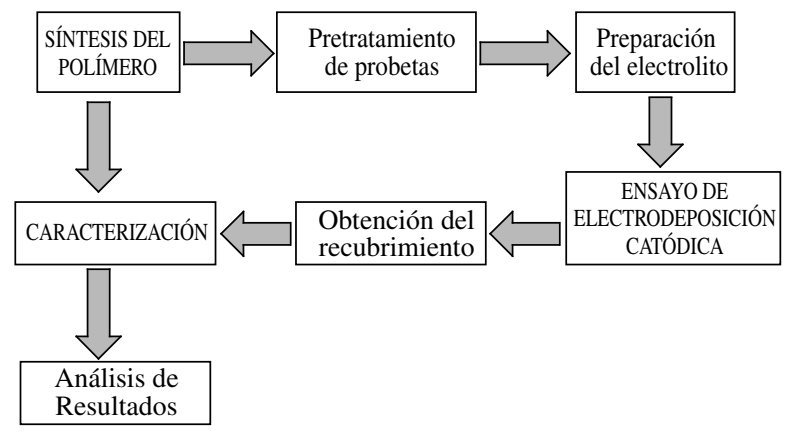

Figura 1. Etapas de la metodología experimental.

\section{Preparación del polímero}

Para esta síntesis se emplearon $400 \mathrm{ml}$ de ácido L-láctico $\left(\mathrm{CH}_{3} \mathrm{CHOHCOOH}\right)$ Carlo Erba de $98 \%$ dentro de un balón de fondo redondo de 3 salidas para un condensador, una entrada de gas y un termómetro. El montaje usado se muestra en la Figura 2.

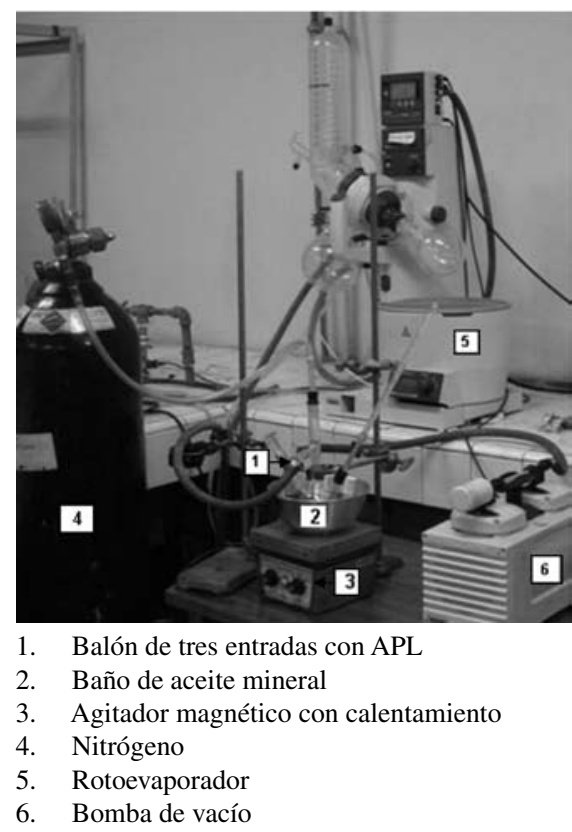

Figura 2. Montaje usado en la síntesis del ácido poli (L-láctico).

La etapa inicial de deshidratación se efectuó mediante calentamiento en un baño de aceite mineral a $100{ }^{\circ} \mathrm{C}$. En la etapa de esterificación se aumentó la temperatura gradualmente a una velocidad de $15^{\circ} \mathrm{C} / \mathrm{min}$ hasta $180^{\circ} \mathrm{C}$. Al llegar a esta condición se adecuó el montaje para imprimirle vacío. Se redujo gradualmente la presión hasta $100 \mathrm{mmHg}$ y se mantuvo la reacción hasta que el agua estuvo presente nuevamente.
Posteriormente, en la etapa de oligomerización, la presión del sistema se llevó hasta $25 \mathrm{mmHg}$ y se continuó bajo estas condiciones durante los diferentes tiempos establecidos. Como catalizador de la reacción se utilizó zinc metálico de alta pureza que se agregó en la etapa final de policondensación con agitación magnética y reducción gradual de la presión hasta $10 \mathrm{mmHg}$. La reacción continuó por 5 horas más bajo estas condiciones, luego de este tiempo se retiró el balón del baño, se envasó el producto y se dejó enfriar.

Durante este procedimiento, se variaron las concentraciones de zinc y el tiempo de reacción (Tabla 1) para obtener cuatro tipos de ácidos polilácticos con propiedades diferentes entre sí, los cuales fueron caracterizados y electrodepositados sobre sustratos de Ti6Al4V.

Tabla 1. Condiciones de síntesis de las muestras de ácido poliláctico.

\begin{tabular}{|c|c|c|}
\hline Muestra & $\begin{array}{c}\text { Tiempo de } \\
\text { reacción } \\
\text { (horas) }\end{array}$ & $\begin{array}{c}\text { Concentración de } \\
\text { catalizador } \\
\left(\% \text { peso } \mathrm{Zn}^{++}\right)\end{array}$ \\
\hline 1 & 20 & 0.5 \\
\hline 2 & 20 & 1.5 \\
\hline 3 & 30 & 0.5 \\
\hline 4 & 30 & 1.5 \\
\hline
\end{tabular}

\section{Caracterización de los polímeros obtenidos}

El producto obtenido se caracterizó mediante espectroscopia de infrarrojo y absorción atómica para verificar la composición del polímero y la presencia de $\mathrm{Zn}^{++}$en su matriz principal. Por análisis de espectroscopia IR se comparó el espectro obtenido con el espectro de ácido poliláctico encontrado en la literatura consultada [10] y posteriormente se compararon los espectros obtenidos para cada una de las condiciones de síntesis para determinar si existió alguna variación en las longitudes de onda de los picos representativos, con el fin de establecer si las variables modificadas durante la polimerización reflejaron algún cambio significativo en la estructura del polímero. Este análisis fue llevado a cabo en un equipo para espectroscopia de infrarrojo FTIR-8400 Shimadzu.

Adicionalmente, las propiedades térmicas de este polímero fueron determinadas con los ensayos de calorimetría diferencial de barrido (DSC) y análisis termogravimétrico (TGA), para lograr una descripción de ellos y poder compararlos. Por medio de estas técnicas, se detallaron las diferentes zonas de degradación presentes en cada uno de los polímeros sintetizados y se compararon sus respectivas temperaturas. 
El TGA fue realizado en una balanza termogravimétrica AT-Instruments 2050, con una velocidad de calentamiento de $10{ }^{\circ} \mathrm{C} / \mathrm{min}$ en un rango de temperatura de 0 a $500{ }^{\circ} \mathrm{C}$, bajo atmósfera de nitrógeno. Las cuatro muestras poliméricas se sometieron a análisis de calorimetría diferencial de barrido (DSC) para determinar su punto de fusión y temperatura de transición vítrea; este análisis fue llevado a cabo en un DSC AT-Instruments.

El ataque químico de los polímeros se efectuó siguiendo la norma ASTM D543-87, con la cual se evaluó su comportamiento para aplicaciones como recubrimientos de implantes temporales. Estos ensayos se hicieron sumergiendo seis muestras de los polímeros obtenidos en dos medios distintos: Solución Ringer $(\mathrm{pH}=7,5)$ a $27^{\circ} \mathrm{C}$ y agua destilada $(\mathrm{pH}=7)$ a $27^{\circ} \mathrm{C}$. El proceso degradativo de estos se evaluó mediante pérdida de masa.

\section{Electrodeposición del polímero}

Los ensayos de electrodeposición del ácido poliláctico obtenido, se realizaron manipulando diferentes variables involucradas en el proceso, con el fin de determinar las condiciones necesarias para obtener un recubrimiento sobre las probetas de Ti6Al4V. Los sustratos en forma de disco de Ti6Al4V de área $3,02 \mathrm{~cm}^{2}$ se prepararon superficialmente de acuerdo a lo antes descrito.

Para realizar la electrodeposición catódica se empleó una celda de vidrio, como se muestra en la Figura 3, con capacidad para $200 \mathrm{ml}$ con dos ánodos de grafito cuya área expuesta fue de $12,57 \mathrm{~cm}^{2}$; la distancia entre ánodo y cátodo fue de $5 \mathrm{~cm}$. Se utilizó una fuente de potencia y una placa de calentamiento con agitación magnética. El electrolito fue preparado a partir de la disolución del ácido poliláctico sintetizado, usando acetona como disolvente en una proporción en volumen de 30:70.

En las pruebas de electrodeposición del polímero se realizó un diseño experimental $2^{5}$ considerando cinco de las variables más importantes del proceso (voltaje aplicado, tiempo, temperatura, agitación en el sistema y las variables de preparación del polímero).

El diseño experimental fue realizado con un software estadístico, el cual arrojó las condiciones para la realización de 36 diferentes pruebas para la obtención de los recubrimientos sobre Ti6Al4V. El montaje utilizado en la electrodeposición se muestra en el Figura 3.

A partir del análisis estadístico de los datos de electrodeposición obtenidos en pruebas preliminares, se definieron las variables empleadas en el diseño experimental. Estas variables y los rangos establecidos se indican en la Tabla 2.
El diseño experimental fue aplicado a las diferentes muestras de polímero, obtenidas con variaciones en el porcentaje en masa de catalizador entre $0,5-1,5 \% \mathrm{Zn}$ y en el tiempo de polimerización entre 20-30 horas. Adicionalmente a algunos de los recubrimientos obtenidos se les realizaron análisis de MEB e infrarrojo para comprobar si las propiedades del polímero se alteraron durante la electrodeposición.

Tabla 2. Variables utilizadas en la electrodeposición de APL sobre Ti6Al4V.

\begin{tabular}{|c|c|c|c|}
\hline Variables & - & o & + \\
\hline Voltaje $(\mathrm{V})$ & 100 & 200 & 300 \\
\hline Tiempo $(\mathrm{min})$ & 4 & 8 & 12 \\
\hline Temperatura $\left({ }^{\circ} \mathrm{C}\right)$ & 25 & 33 & 40 \\
\hline Agitación $(\mathrm{rpm})$ & 0 & 200 & 400 \\
\hline
\end{tabular}
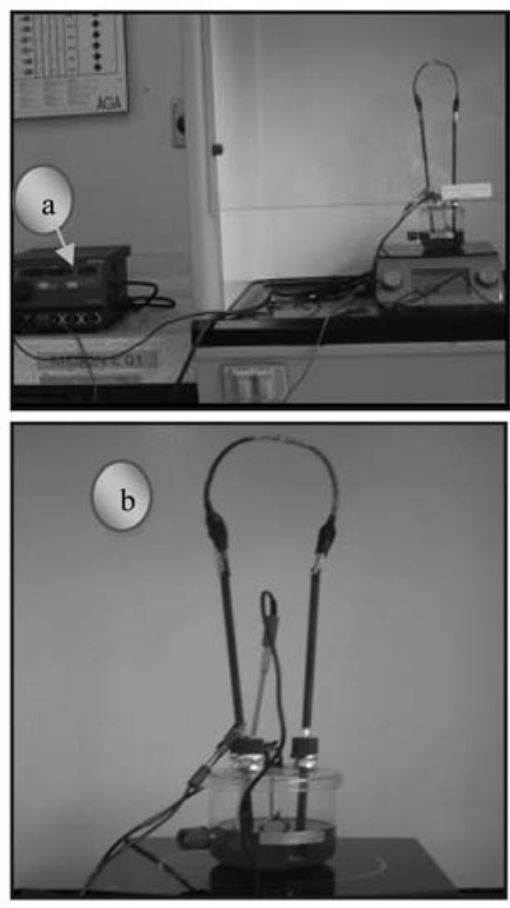

Figura 3. Montaje utilizado en la electrodeposición del ácido poliláctico. Fuente de potencia (a), detalle de la celda empleada (b).

\section{Caracterización del recubrimiento polimérico obtenido}

Luego de la electrodeposición, las probetas fueron secadas con aire frío en flujo laminar para eliminar los excesos de solvente, luego fueron pesadas y analizadas. Se realizó el análisis del recubrimiento mediante ataque químico, siguiendo el mismo procedimiento utilizado para las muestras poliméricas, sumergiendo dos de las probetas recubiertas en soluciones de iguales características. 
Como resultado del análisis estadístico realizado se escogieron ocho recubrimientos que correspondieron a las condiciones de electrodeposición más influyentes. Para evaluar la morfología de los recubrimientos obtenidos se evaluaron las superficies mediante análisis de microscopia óptica, en la cual se pudo observar la homogeneidad del recubrimiento y del sustrato, como la aparición de pequeños poros en el sustrato metálico y la adherencia del recubrimiento, además se llevó a cabo la determinación del espesor óptico de cada uno.

\section{RESULTADOS Y DISCUSIÓN}

El termograma de la Figura 4 se utilizó para establecer los rangos de temperatura de trabajo durante la polimerización del ácido láctico. Este reveló una pérdida de masa entre $127-185^{\circ} \mathrm{C}$ y una degradación total del mismo por encima de $200^{\circ} \mathrm{C}$. Estos resultados ayudaron a consolidar las bases para llevar a cabo el proceso de polimerización propuesto.

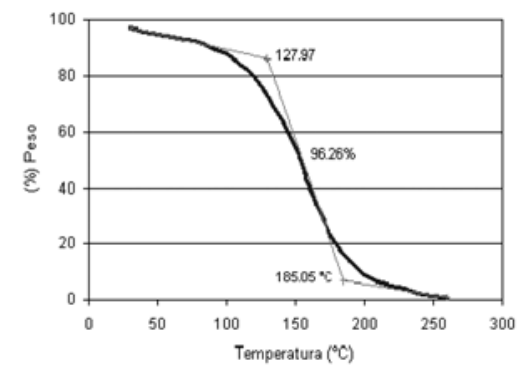

Figura 4. Termograma para el ácido L-láctico realizado por TGA.

\section{Polimerización del ácido láctico y caracterización del polímero obtenido}

Durante los procesos de policondensación, se observó la aparición de un producto intermedio en forma de cristales translúcidos, el cual fue caracterizado por espectroscopia de infrarrojo, determinando la presencia del dímero cíclico del ácido láctico. Este producto comenzó a aparecer al cabo de la novena hora de reacción y estuvo presente hasta el final de la misma, ubicándose en las ramificaciones de las conexiones de vidrio en el montaje de síntesis del polímero.

\section{Microscopia electrónica de barrido y espectroscopia dispersiva de energía (SEM-EDS)}

Mediante este análisis se comprobó la presencia de zinc en la matriz principal del polímero obtenido en la Figura 5, se aprecia este elemento a una longitud de onda de 8,4 con sus respectivas familias a bajas longitudes de onda. La incorporación de $\mathrm{Zn}^{++}$dentro de la cadena polimérica favoreció la electrodeposición catódica del polímero.
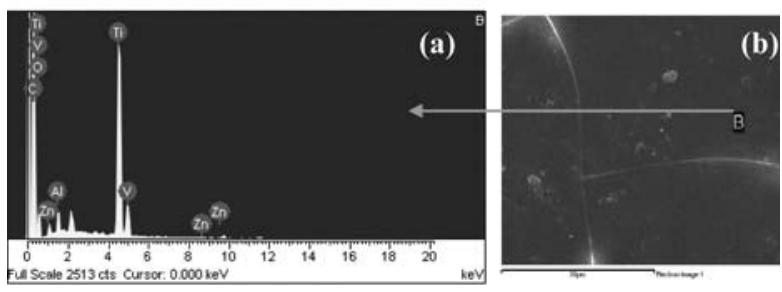

Figura 5. Espectro EDE -\% atómico vs $\mathrm{keV}$ (a) y micrografía MEB (b) a partir de la superficie del polímero de APL obtenido.

\section{Análisis por espectroscopia de infrarrojo IR}

Luego de comparar el espectro infrarrojo de transformada de Fourier FTIR del material polimérico obtenido en una prueba inicial con el espectro para el ácido poliláctico (APL) encontrado en la literatura [11] pudo comprobarse que el polímero sintetizado fue similar al APL reportado debido a la similitud de los picos observados. El espectro del material obtenido reveló la absorción característica del APL; el pico que se presenta en $1.751 \mathrm{~cm}^{-1}$ indica la presencia del grupo $\mathrm{C}=\mathrm{O}$. Otro pico en $3.000 \mathrm{~cm}^{-1}$ es debido al grupo $\mathrm{C}-\mathrm{H}$ y entre 1.000 y $1.200 \mathrm{~cm}^{-1} \mathrm{se}$ encuentra otro pico sobresaliente que indica la presencia de un enlace C-C en el polímero.

Adicionalmente, se realizaron análisis por espectroscopia de infrarrojo a los polímeros obtenidos bajo las distintas condiciones de polimerización, para comparar por medio de sus espectros si existió alguna variación en las longitudes de onda de los picos representativos, con el fin de determinar si las variables modificadas durante la polimerización reflejaron algún cambio significativo en la estructura del polímero. Los resultados de este análisis (Figura 6) mostraron que los cuatro polímeros presentaron los mismos picos de absorción que presentó el polímero obtenido en la prueba inicial, lo que evidenció que en su composición final no tiene relevancia alguna la modificación del tiempo de polimerización y el porcentaje de catalizador durante la síntesis. 


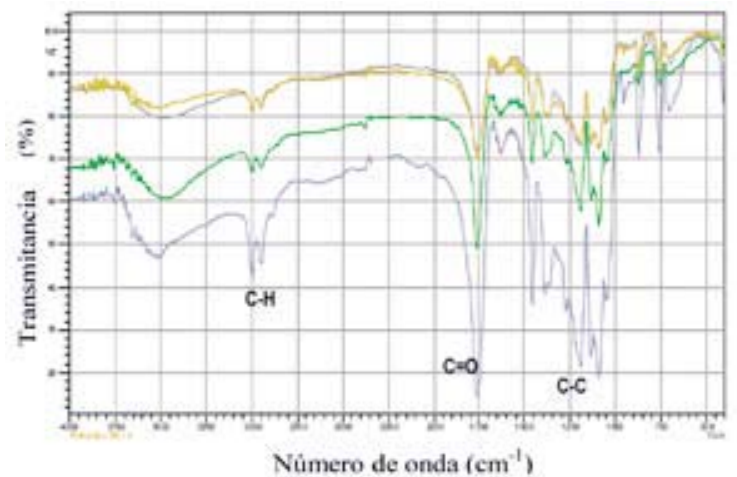

Figura 6. Diagramas comparativos de los polímeros obtenidos a partir del espectro infrarrojo de transformada de fourier (FTIR).

\section{Análisis térmico}

A continuación se presentan los termogramas de la prueba de calorimetría diferencial de barrido (DSC) de los polímeros obtenidos. Éstos muestran la temperatura de transición vítrea en aproximadamente $42{ }^{\circ} \mathrm{C}$, la cual es menor que la reportada para el APL [2-3]. Posiblemente se explique esto por la diferencia en el peso molecular. También se observó la temperatura de degradación en aproximadamente $335^{\circ} \mathrm{C}$ (Figura 7).

Los cuatro polímeros obtenidos muestran un comportamiento térmico similar, lo cual revela que las modificaciones realizadas en la polimerización no afectan significativamente sus propiedades térmicas.

Los picos de fusión son exotérmicos y relativamente anchos; este factor indica una variabilidad en las temperaturas de fusión debido a lo disperso de los pesos moleculares de las muestras poliméricas. La presencia de discontinuidades en la línea base después de $\sim 330^{\circ} \mathrm{C}$ puede ser un indicio de la descomposición de los polímeros, la cual fue corroborada mediante análisis termogravimétricos.

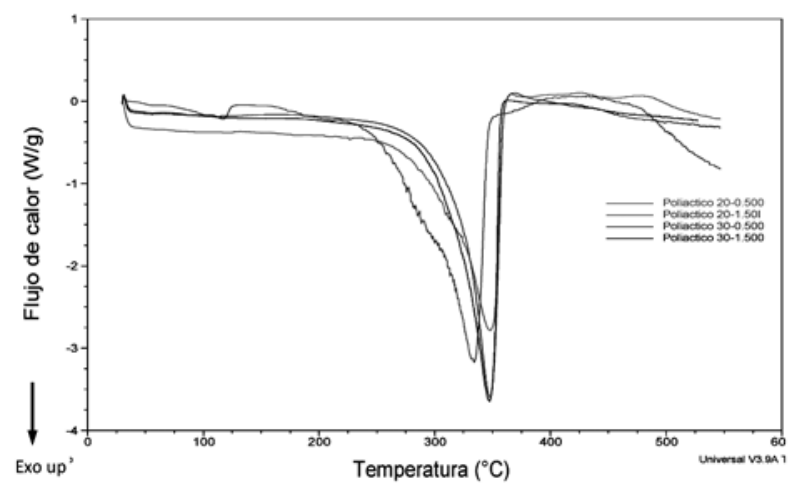

Figura 7. Termogramas DSC de las muestras de polímero obtenidas.

\section{Análisis termogravimétrico TGA}

Como se puede observar en la Figura 8, las variaciones realizadas en la preparación del polímero (tiempo de reacción y \% de catalizador) influyen en la degradación térmica, notándose su diferencia en el desplazamiento de las temperaturas de degradación.

Los polímeros correspondientes al mayor número de horas de polimerización presentan una curva de degradación que se desplaza hacia la derecha del termograma, lo que significa que son un poco más estables térmicamente.

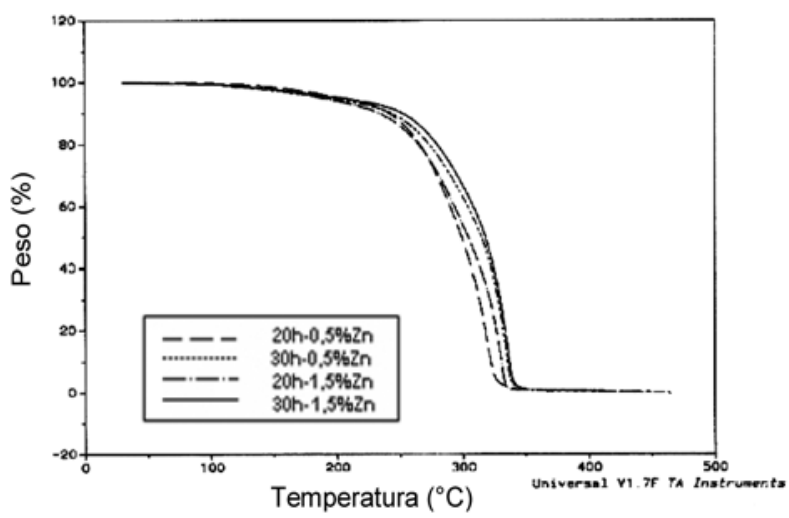

Figura 8. Termogramas TGA de las muestras de polímero obtenidas.

El análisis para los cuatro polímeros presenta tres diferentes zonas que fueron analizadas determinando sus distintos rangos de temperatura en la Tabla 3. En la primera zona hay una pérdida en masa del material, que corresponde a pérdidas de humedad, compuestos de peso molecular promedio y la volatilización de las ramificaciones presentes en las moléculas que se encuentran formando los enlaces.

La segunda zona corresponde a la degradación térmica o el rompimiento del entrecruzamiento de los enlaces. En la tercera zona se da la completa descomposición de las redes en $\sim 330^{\circ} \mathrm{C}$, lo que confirmó que los picos sobresalientes presentados en el DSC corresponden a la temperatura de degradación de los polímeros.

Tabla 3. Rangos de temperatura de descomposición de los polímeros obtenidos.

\begin{tabular}{|c|c|c|c|}
\hline Muestra & $\begin{array}{c}\text { Zona 1 } \\
\text { Pérdida de } \\
\text { humedad }\left({ }^{\circ} \mathrm{C}\right)\end{array}$ & $\begin{array}{c}\text { Zona 2 } \\
\text { Degradación } \\
\text { Térmica }\left({ }^{\circ} \mathrm{C}\right)\end{array}$ & $\begin{array}{c}\text { Zona 3 } \\
\text { Degradación total } \\
\text { del polímero }\left({ }^{\circ} \mathrm{C}\right)\end{array}$ \\
\hline 1 & $91.71-243.51$ & $243.51-325.18$ & $325.18-400$ \\
\hline 2 & $125.10-248.53$ & $248.53-331.84$ & $331.84-400$ \\
\hline 3 & $150.59-246.84$ & $246.84-337.10$ & $337.10-400$ \\
\hline 4 & $198.26-250.26$ & $250.26-339.51$ & $339.51-400$ \\
\hline
\end{tabular}




\section{Ataque químico}

En la Tabla 4 se presentan los valores de pérdida de masa de los polímeros luego de permanecer expuestos durante una semana en dos soluciones diferentes.

La comparación de resultados permite observar cómo varió la degradación en función de la composición del polímero y del tiempo de polimerización.

Tabla 4. Resultados prueba de ataque químico para muestras de polímero.

\begin{tabular}{|c|c|c|c|c|c|}
\hline Muestra & Solución* & $\begin{array}{c}\text { Masa } \\
\text { inicial } \\
(\mathrm{g})\end{array}$ & $\begin{array}{c}\text { Masa } \\
\text { final } \\
(\mathrm{g})\end{array}$ & $\begin{array}{c}\text { Diferencia } \\
(\mathrm{g})\end{array}$ & $\begin{array}{c}\text { Variación } \\
\text { masa } \\
\%\end{array}$ \\
\hline 2 & $\mathrm{~A}$ & 0.7953 & 0.7919 & 0.0034 & 0.43 \\
\hline 3 & $\mathrm{~A}$ & 1.3882 & 1.3873 & 0.0009 & 0.065 \\
\hline 4 & $\mathrm{~A}$ & 0,1651 & 0.1644 & 0.0007 & 0.42 \\
\hline 2 & $\mathrm{~B}$ & 1.1495 & 1.1259 & 0.0236 & 2.05 \\
\hline 3 & $\mathrm{~B}$ & 1.3648 & 1.3625 & 0.0023 & 0.17 \\
\hline 4 & $\mathrm{~B}$ & 0.8678 & 0.8673 & 0.0005 & 0.058 \\
\hline
\end{tabular}

* Solución A: Agua destilada.

Solución B: Solución Ringer.

En la superficie de todas las muestras se observó la aparición de algunos poros y grietas, así como un color blanco, mientras que en su interior aún conservaban su color original. También se presentó hinchamiento en todas las muestras, resultados que mostraron que el grado de hinchamiento fue menor en las muestras con 20 horas de polimerización.

\section{Electrodeposición catódica}

El análisis de los datos se llevó a cabo mediante un software estadístico, introduciendo como variable respuesta el porcentaje de ganancia en masa de las probetas recubiertas. Se observó la influencia de cada una de las variables involucradas en el proceso de electrodeposición.

La variable más significativa fue la agitación, seguida de la interacción entre el tiempo y la temperatura. Sin embargo, la temperatura por sí sola no tuvo mayor influencia. El modelo estadístico resultante para la variable de respuesta (ganancia de masa) es:

\section{Ganancia de masa $=3.76917+0.433438 *$ Agitación $-0.413438 *$ Temperatura $*$ Tiempo}

En la Figura 9 se presenta la influencia del tiempo de electrodeposición sobre la ganancia en masa de las probetas recubiertas.

Como se observa en la Figura 9, a menores tiempos de electrodeposición y a mayores temperaturas, se presenta mayor ganancia de masa. Caso contrario sucede a mayores tiempos de electrodeposición donde a menor temperatura se presenta mayor ganancia de masa. Este hecho mostró un mejor panorama del efecto que causa la relación temperatura/tiempo en el proceso de electrodeposición catódica que se utilizó para obtener los recubrimientos.

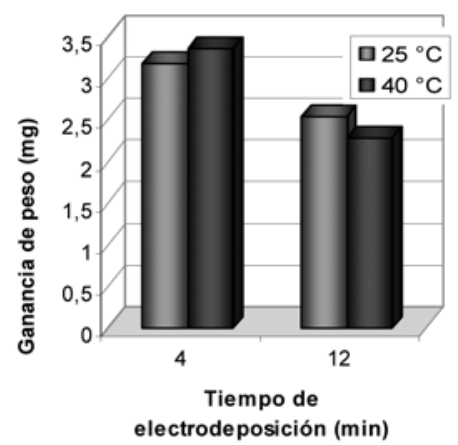

Figura 9. Relación tiempo/temperatura sobre la ganancia en masa de las probetas de Ti6Al4V recubiertas.

\section{Caracterización del recubrimiento polimérico}

El resultado de la electrodeposición catódica fue un recubrimiento de ácido poli (L-láctico) semitransparente como el que se presenta en la Figura 10.

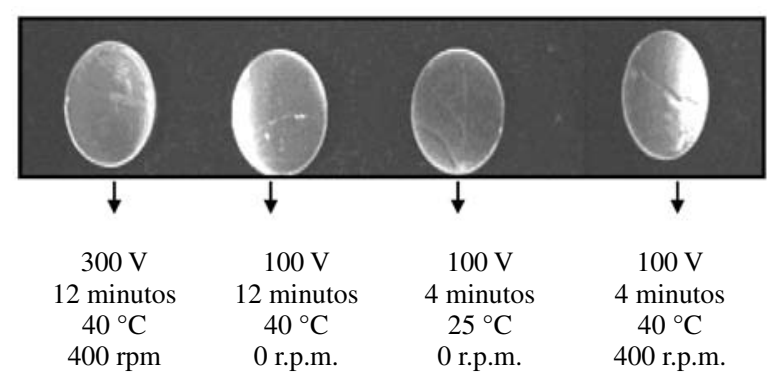

Figura 10. Recubrimientos sobre probetas de Ti6Al4V.

\section{Ganancia en masa}

Esta fue la variable empleada como respuesta para la evaluación del recubrimiento con ayuda de un software estadístico. Los ensayos realizados con el polímero durante 20 horas presentaron menores ganancias en masa. La Figura 11a muestra que esta variable se ve directamente afectada por la agitación en los ensayos de electrodeposición realizados con el polímero de menor tiempo de síntesis (20 horas) y que además, el efecto de la temperatura no es significativo bajo estas condiciones de voltaje y tiempo. Por el contrario, la mayor velocidad de agitación (400 rpm) mayor influencia frente a la electrodeposición de poli-L-láctico sobre Ti6Al4V. 
Como se observa en la Figura 11b, mayor masa de polímero depositado se presentó a $25^{\circ} \mathrm{C}$ y 300 voltios, sin embargo a $40{ }^{\circ} \mathrm{C}$ se presentó mayor ganancia de masa a 100 voltios. Sin olvidar la cinética de deposición, a mayores voltajes se necesitó menos tiempo que el necesario a bajos voltajes para depositar el polímero. Además se observa que la mayor ganancia en masa se obtuvo para la probeta número 25 , para la cual las condiciones fueron: $100 \mathrm{~V}, 12$ minutos, $25^{\circ} \mathrm{C}, 400 \mathrm{rpm}, 30 \mathrm{~h}, 1,5$.

\section{a. $300 \mathrm{~V}, 4$ minutos}

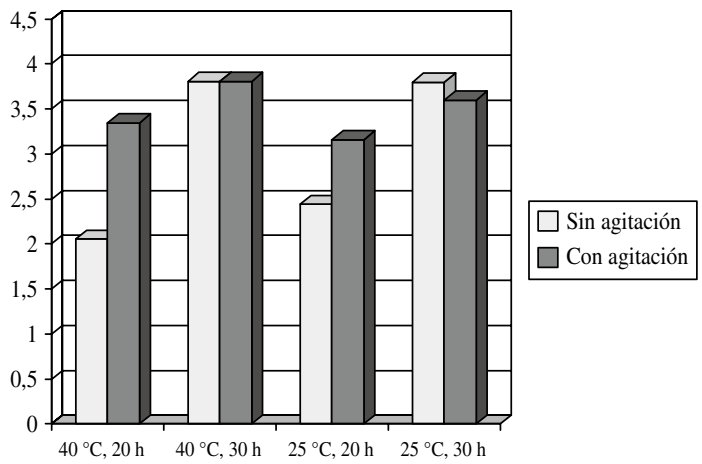

b. $400 \mathrm{rpm}, 20 \mathrm{~h}, 1,5 \%$

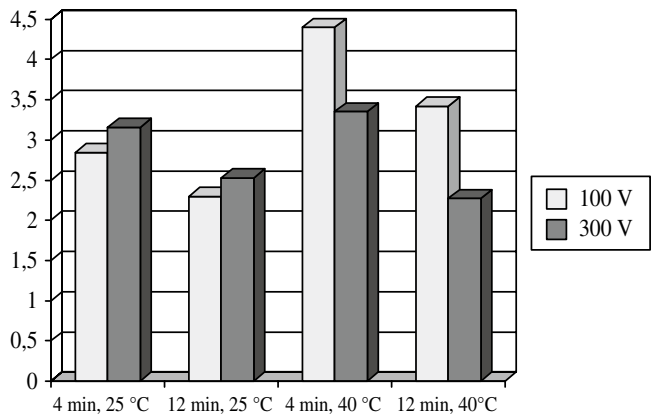

Figura 11. Efecto de las variables de electrodeposición en la ganancia de masa. a. $300 \mathrm{~V}, 4$ minutos, b. $400 \mathrm{rpm}, 20 \mathrm{~h}, 1,5 \%$.

\section{Ataque químico}

En la Tabla 5 se presentan los resultados de la variación en porcentaje de masa de dos de las probetas recubiertas, luego de una semana de ser sometidas al ataque químico en dos medios distintos.

Tabla 5. Resultados prueba de ataque químico para muestras de recubrimiento polimérico.

\begin{tabular}{|c|c|c|c|c|c|}
\hline $\begin{array}{c}\text { Probetas de } \\
\text { Ti6Al4V } \\
\text { recubiertas }\end{array}$ & Solución & $\begin{array}{c}\text { Masa } \\
\text { inicial } \\
(\mathrm{mg})\end{array}$ & $\begin{array}{c}\text { Masa final } \\
(\mathrm{mg})\end{array}$ & $\begin{array}{c}\text { Diferencia } \\
\text { en masa } \\
(\mathrm{mg})\end{array}$ & $\begin{array}{c}\text { Variación } \\
\text { masa \% }\end{array}$ \\
\hline $\begin{array}{c}30 \mathrm{~h}- \\
1.5 \% \mathrm{Zn}\end{array}$ & $\begin{array}{c}\text { Agua } \\
\text { destilada }\end{array}$ & 1.01158 & 1.01146 & 0.00012 & 0.012 \\
\hline $\begin{array}{c}30 \mathrm{~h}- \\
1.5 \% \mathrm{Zn}\end{array}$ & $\begin{array}{c}\text { Solución } \\
\text { Ringer }\end{array}$ & 1.01287 & 1.01241 & 0.00046 & 0.045 \\
\hline
\end{tabular}

Los recubrimientos presentaron un color blanco durante unos días luego de haber sido sumergidos en las soluciones. Según los valores de porcentaje de variación de masa, se puede afirmar, que la degradación de la probeta sumergida en la solución Ringer se realizó más rápidamente ya que ésta, después del ataque químico, presentó mayor diferencia de masa.

Se observó que el recubrimiento en esta solución desapareció casi por completo a excepción de algunas partes con espesores mayores, mientras que el recubrimiento de la probeta que se encontraba sumergida en el agua destilada, se conservó casi en su totalidad.

\section{Microscopia óptica}

Las imágenes de micrografías con ampliación óptica de 100x (Figura 12) muestran la morfología superficial de cuatro recubrimientos obtenidos a diferentes condiciones de electrodeposición, lo cual evidencia la variación de la homogeneidad de estos bajo condiciones de electrodeposición diferentes, además muestra la influencia del pretratamiento de las probetas de Ti6Al4V en la calidad del recubrimiento.

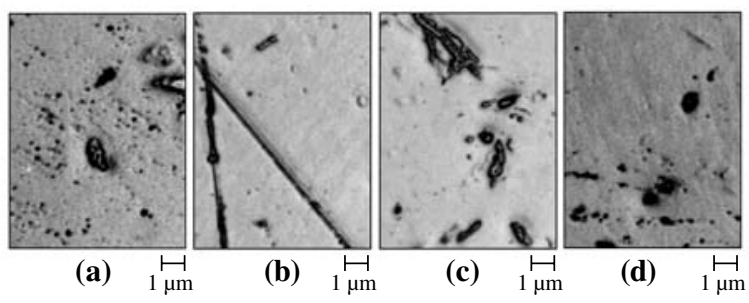

Figura 12. Imágenes de la superficie de los recubrimientos obtenidos.

Este análisis permitió observar las imperfecciones de los recubrimientos, relacionados con los de las probetas de Ti6Al4V, y con estos resultados, se logró realizar un análisis comparativo de estos, para ser caracterizados.

Las probetas escogidas para realizar las pruebas de microscopia óptica fueron las que obtuvieron una mayor ganancia de masa luego de la electrodeposición. Los cuatro recubrimientos mostrados en la Figura 12 se realizaron con temperatura de electrodeposición igual a $40{ }^{\circ} \mathrm{C}$ y el material polimérico sintetizado con un tiempo de policondensación de 30 horas y un porcentaje de catalizador de zinc de $1,5 \%$. Se pudo corroborar que la agitación de la solución polimérica en el momento de la electrodeposición fue determinante en la homogeneidad superficial, presentándose mayor porosidad en el recubrimiento de la Figura 12a, el cual se realizó sin agitación, comparado con las Figuras $12 \mathrm{~b}$, $12 \mathrm{c}, 12 \mathrm{~d}$ correspondientes a recubrimientos realizados bajo agitación de $400 \mathrm{rpm}$. 
La Tabla 6 muestra los resultados de espesor óptico para ocho de las probetas que corresponden a las condiciones más influyentes de electrodeposición según el análisis estadístico realizado. Las ocho probetas escogidas para evaluar calidad del recubrimiento y que fueron electrodepositadas según las condiciones consignadas en la Tabla 6, presentaron diferencias en su espesor óptico. Estas diferencias fueron estudiadas y relacionadas con la ganancia en masa, la cual fue la primera variable de respuesta tenida en cuenta para la caracterización.
En el análisis de microscopia de las probetas se observó que aquellas que presentaban defectos, rugosidad y huecos en la superficie metálica revelaron valores más altos en la ganancia de masa y valores bajos en el espesor óptico, esto se explica porque durante el tiempo de electrodeposición el polímero se deposita primero entre los defectos del material y luego sí se desplaza sobre la superficie para lograr el recubrimiento. Este tipo de comportamiento en los recubrimientos obtenidos garantizó la adherencia del APL sobre el Ti6Al4V.

Tabla 6. Espesores ópticos de las probetas escogidas ( $\mathrm{V}=300 \mathrm{~V}$, condiciones de polímero $30 \mathrm{~h}-1.5 \% \mathrm{Zn})$.

\begin{tabular}{|c|c|c|c|c|c|}
\hline \multirow[b]{2}{*}{ \# Probeta } & \multicolumn{3}{|c|}{ Condiciones de electrodeposición } & \multirow{2}{*}{$\begin{array}{l}\text { Ganancia de } \\
\text { masa (mg) }\end{array}$} & \multirow{2}{*}{$\begin{array}{c}\text { Espesor } \\
\text { optico }(\mu \mathrm{m})\end{array}$} \\
\hline & $\begin{array}{c}\text { Tiempo } \\
\text { (minutos) }\end{array}$ & $\begin{array}{c}\text { Temperatura } \\
\left({ }^{\circ} \mathbf{C}\right)\end{array}$ & $\begin{array}{c}\text { Agitación } \\
\text { (rpm) }\end{array}$ & & \\
\hline 22 & 12 & 25 & 0 & 4 & 2.82 \\
\hline 23 & 4 & 25 & 0 & 3.8 & 3.16 \\
\hline 26 & 4 & 25 & 400 & 3.6 & 2.72 \\
\hline 27 & 12 & 25 & 400 & 4.55 & 2.95 \\
\hline 29 & 4 & 40 & 0 & 3.81 & 2.80 \\
\hline 31 & 12 & 40 & 0 & 4.72 & 3.81 \\
\hline 33 & 12 & 40 & 400 & 4.73 & 3.57 \\
\hline 35 & 4 & 40 & 400 & 3.81 & 1.90 \\
\hline
\end{tabular}

\section{CONCLUSIONES}

Las condiciones de síntesis que aportaron mejores propiedades a los polímeros obtenidos y mejoraron, tanto el comportamiento en la electrodeposición como la calidad del recubrimiento, fueron 30 horas de polimerización y adición de $1,5 \%$ en peso de catalizador de zinc metálico.

El material polimérico obtenido por policondensación de ácido L-láctico, utilizando zinc metálico bajo las condiciones descritas en este trabajo, es ácido poli (L-láctico), y tiene las propiedades eléctricas necesarias para ser electrodepositado sobre sustratos de Ti6Al4V utilizando técnicas de electrodeposición catódica.

Para el rango de temperatura entre $25^{\circ} \mathrm{C}-50{ }^{\circ} \mathrm{C}$ no se presentan variaciones importantes en la degradación térmica, por lo cual el material podría ser utilizado en el recubrimiento de implantes ortopédicos.

\section{AGRADECIMIENTOS}

Los autores agradecen a la ingeniera Pilar González por sus valiosos aportes en esta investigación y a COLCIENCIAS por su apoyo financiero.

\section{REFERENCIAS}

[1] W. Ciccone, C. Motz and J. Bentley. "Bioabsorbable Implants in Orthopaedics: New Developments and Clinical Applications". Journal of the American Academy of Orthopaedic Surgeons. Vol. 9, pp. 280288. 2001.

[2] W.H. Carothers, G.L. Dorough and F.J. Van Natta. "Studies of Polymerization and Ring Formation. The Reversible Polymerization of Six-Membered Cyclic Esters". Journal of the American Chemical Society. Vol. 54, pp. 761-772. 1932.

[3] E. Gallardo, J. San Román y A. López. "Polímeros biodegradables en medicina: diseño estructural y desarrollo de materiales". Revista de Plásticos Modernos. Vol. 77, p. 511. 1999.

[4] Kerrzo and Conroy. "Electrochemical studies on the stability and corrosion resistance of titaniumbased implant materials". Biomaterials. Vol. 21, pp. 2103-2113. 2000.

[5] T. Hayashi. "Biodegradable polymers for biomedical uses”. Prog. Polym. Sci. Vol. 19, pp. 663-702. 1994. 
[6] Kulkarni R., R.G. Moore and A.F. Hegyeli. "Biodegradable Poly (lactic acid) Polymers". Journal of Biomedical Materials Research. Vol. 5, pp. 169-181. 1971.

[7] W.S. Drumond, S. H. Wang e C.G. Mothé. "Síntese e caracterização do copolímero poli (Ácido lático-bglicol etilênico)". Polímeros: Ciência e tecnologia. Vol. 14, pp. 74-79. 2004.

[8] G. Schwach, J. Coudane, R. Engel and M. Vert. "Ring Opening Polymerization of D, L-Lactide in the Presence of Zinc Metal and Zinc Lactate". Polymer International. Vol. 46, pp. 177-182. 1998.

[9] G. Schwach, J. Coudane, R. Engel and M. Vert. "More about the polymerization of lactides in the presence of stannous octoate". Journal Of Polymer Science. Vol. 35, pp. 3431-3440. 1997.

[10] Gonga Q., Wang, Li-Qun and Tu K. "In situ polymerization of starch with lactic acid in aqueous solution and the microstructure characterization". Carbohydrate Polymers. Vol. 64, pp. 501-509. 2006.

[11] Yumei Xiao, Dongxiao Li, Xuening Chen, Jian Lu, Hongsong Fan and Xingdong Zhang. "Preparation and cytocompatibility of chitosan-modified polylactide". Journal of Applied Polymer Science. Vol. 110, Issue 1, pp. 408-412. October 2008.

[12] J. Pan, M. Zhao, Y. Liu, B. Wang and L. Yang. "Development of a new poly (ethylene glycol)-graftpoly (D,L-lactic acid) as potential drug carriers". Journal of Biomedical Materials Research Part A. Vol. 89A, Issue 1, pp. 160-167. 2009.

[13] H. Zhao, K. Saatchi and U.O. Häfeli. "Preparation of biodegradable magnetic microspheres with poly (lactic acid)-coated magnetite". Journal of Magnetism and Magnetic Materials. Vol. 321, Issue 10, pp. 1356-1363. 2009.

[14] H.T. Oyama. "Super-tough poly (lactic acid) materials: Reactive blending with ethylene copolymer" Polymer. Vol. 50, Issue 3, pp. 747-751. Enero 2009.

[15] Yan-Bing Luo, Wen-Da Li, Xiu-Li Wang, Da-Yun $\mathrm{Xu}$ and Yu-Zhong Wang. "Preparation and properties of nanocomposites based on poly (lactic acid) and functionalized $\mathrm{TiO}_{2}$ ". Acta Materialia. Vol. 57, Issue 11, pp. 3182-3191. Junio 2009.

[16] Yoshiyuki Nakano, Yoshio Hori, Akira Sato, Tetsuo Watanabe, Shuji Takada, Hitoshi Goto, Akiko Inagaki, Yoshito Ikada and Susumu Satomi. "Evaluation of a Poly (1-lactic acid) Stent for Sutureless Vascular Anastomosis". Annals of Vascular Surgery. Vol. 23, Issue 2, pp. 231-238. March 2009.

[17] M.E. Navarro. "Desarrollo y Caracterización de Materiales Biodegradables para Regeneración Ósea". Memoria de tesis presentada para optar al grado de Doctor en Ciencias. Especialidad Ingeniería Química por la Universitat Politècnica de Catalunya. 2005.

[18] R. Camargo. "Estudio de la Electropolimerización de pirrol en presencia de PS, PSS y SBR". Trabajo de Postgrado, Escuela de Ingeniería Química, Universidad Industrial de Santander; Bucaramanga. 1999.

[19] F. Machuca. "Estudio del proceso de electrodeposición de emulsiones poliméricas caso de estudio (Estireno-Butadieno)". Trabajo de maestría, Escuela de Ingeniería Química. Universidad Industrial de Santander. Bucaramanga. 1997. 